\title{
Ultracompliant thermal probe array for scanning non-planar surfaces without force feedback
}

\author{
Shamus McNamara, Amar S Basu, JooHyung Lee \\ and Yogesh B Gianchandani \\ Department of Electrical Engineering and Computer Science, University of Michigan, \\ Ann Arbor, MI 48109-2122, USA \\ E-mail: yogesh@umich.edu
}

Received 28 June 2004, in final form 11 October 2004

Published 29 October 2004

Online at stacks.iop.org/JMM/15/237

\begin{abstract}
This paper describes an array of micromachined thermal probes for scanning thermal microscopy for which the structural design and choice of materials virtually eliminate the need for $\mathrm{z}$-axis mechanical feedback in contact mode scans. The high mechanical compliance accommodates significant topographical variation in the sample surface and prevents damage to soft samples. Thin film metal bolometers are molded into tips at the end of each cantilever in the array, and are sandwiched between two layers of polyimide that serves as the structural material. The probes overhang a Si substrate on which they are fabricated. Since integrated actuators and accompanying circuitry are no longer required, the prospect of scaling from the present eight-probe version to large numbers of probes for high speed, high resolution thermal mapping of large areas with simple detection circuitry is enhanced. The scalability and performance of the eight-probe prototype are evaluated, addressing issues of speed versus resolution, and thermal and mechanical decoupling. The results demonstrate that contact mode scans can provide better than $2 \mu \mathrm{m}$ spatial resolution at speeds greater than $200 \mu \mathrm{m} \mathrm{s}^{-1}$ and show a 6.5 bit topographical resolution over a $7 \mu \mathrm{m}$ dynamic range. Line scans obtained with a single-shank probe suggest that there are good prospects of obtaining images showing a lateral spatial resolution of less than $50 \mathrm{~nm}$.
\end{abstract}

\section{Introduction}

First introduced in 1986 [1], scanning thermal microscopy (SThM) has found a unique place among the various techniques for high resolution scanning microscopy. It permits mapping of topography, temperature, thermal conductivity, thermal capacitance and performing micro-calorimetry with sub-50 nm spatial resolution [2, 3]. These functions cannot typically be performed by optical imaging methods, which are additionally limited by infrared wavelength to spatial resolution of 10-12 $\mu \mathrm{m}$. A number of applications can be envisioned for tools with spatial resolution in the 0.05-5 $\mu \mathrm{m}$ range, such as biomedical diagnostics or cellular mapping, semiconductor process monitoring for sub-surface defects, and semiconductor device diagnostics such as failure analysis by hot-spot detection. The scanning probe has a thermal sensor (typically a bolometer or thermocouple) in the vicinity of a sharp tip located at the end of a cantilever. Scanning is best performed with the probe in contact with the sample to eliminate the high thermal resistance of an air gap, but this conventionally requires a mechanical feedback loop to prevent the probe from scratching the sample. Similar to an atomic force microscope (AFM), the contact force is sensed by measuring probe deflection with a reflected laser. The contact pressure of the probe is adjusted using a positioner that is controlled by the error signal from the laser detector. This signal also provides a measure of the topographical variations in the sample independently of the variations in temperature or thermal properties. 
In the case of the bolometer (or thermistor) probe the thermal bias between the probe and the sample is provided by the resistor that also serves as a sensor. Heat flows from this resistor at the probe tip through the sample to the supporting substrate, which is nominally at a fixed temperature. As the probe is rastered across the sample surface, variations in temperature, thermal properties or physical properties that affect thermal conductance of the sample can be sensed by the resistor. These variations are mapped in one of two ways: applying constant power to the resistor and monitoring the changes in its resistance (which is indicative of its temperature), or using a servo-controlled circuit to maintain the resistor at a constant temperature and monitoring the variations in power required to do this. For a passive sample that is at constant temperature, this image can contain information related to variations in both topography and thermal properties. For some applications this information does not need to be separated, particularly if the either one of the two variables is not changing significantly. In cases that require the thermal information to be separated, this can be done by subtracting the topographic image, obtained as described previously, from the composite image.

The desire to increase throughput in scanning probe microscopy (both SThM and AFM) has prompted the design of arrays in which multiple probe tips scan in parallel [4-9]. The mechanical feedback loop in a conventional SThM can be either at the chip level, causing the entire array to be raised and lowered, or at the probe level. For data storage applications, in which the topographical variation is only a few tens of nanometers, chip level feedback may be sufficient, as was shown by the first large scale $(32 \times 32)$ thermal probe array, IBM's Millipede [4]. However, for general purpose scientific applications, the local topographical variations can be in excess of $1 \mu \mathrm{m}$, and each thermal probe must be able to deflect independent of the other probes in order to follow the surface. This is best performed by individual feedback for each tip. As the array size is increased, however, this rapidly becomes a difficult issue because parallel scanning requires each cantilever to have its own addressable feedback loop. Individual actuation of cantilevers has been explored using piezoelectric films [5, 6], electrostatic actuators [7] and electrothermal bimorphs [8, 9], for example. While these approaches are effective for limited variations in topography, they do not easily accommodate samples with micrometer scale topographical variation, such as integrated circuits or biological cells. Furthermore, all methods of integrating actuators on the thermal probe require additional fabrication steps, control circuitry and electrical interconnect, which greatly increase the manufacturing and calibration complexity.

In this work ${ }^{1}$ we present an ultracompliant probe array for use in thermal imaging that can be scanned across a sample with minimal contact force and without mechanical feedback. The use of polyimide as the cantilever structural material, previously reported by our group, offers not only a high thermal isolation, but a very high compliance [3], resulting in a $10-100 \times$ reduction in contact force over conventional scanning probes. The contact force for each cantilever in the array remains low over a wide range of deflections, eliminating the need for probe level feedback that is typically required to

\footnotetext{
1 Portions of this paper have appeared in conference abstract form in [10].
}

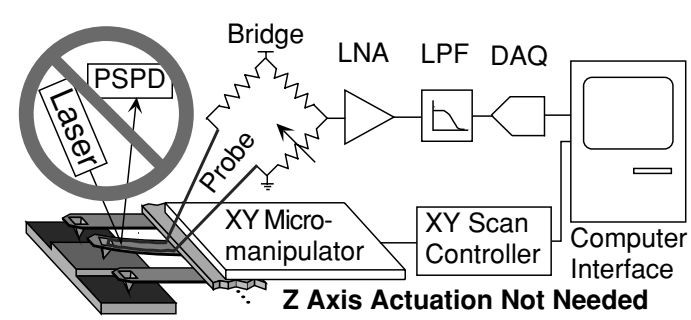

Figure 1. Z-axis feedback and integrated actuators are not required in the ultracompliant thermal probe array presented here. A simple open loop interface circuit is sufficient to both bias the probe tip temperature and read the tip temperature via changes in probe resistance.

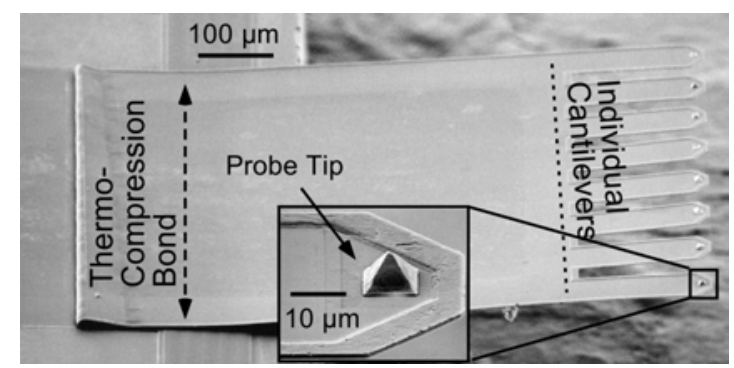

Figure 2. SEM of the thermal probe array after assembly with an inset showing the metal probe tip. Individual cantilevers protrude from a common beam segment. The entire array is flipped over and held in place in one step.

prevent damage to the sample and the tip. In addition, the structure (figure 1) can be designed in a manner such that the cantilevers are decoupled both thermally and mechanically, enabling high-speed parallel thermal mapping of samples with large topographical variation using simple detection circuitry and no integrated actuators. A dynamic range of $>5 \mu \mathrm{m}$ is targeted to permit use in several target applications including thermal analysis of integrated circuits, profiling of surface micromachined MEMS and thermal characterization of irregular polymer samples.

\section{Device structure and operation}

A fabricated thermal probe array is shown in figure 2. Eight individual cantilevers extend off a common beam segment overhanging from the edge of the die. At the end of each cantilever is a pyramid-shaped thermal probe tip extending out from the cantilever comprising a thin film metal resistor. The cantilevers and shank are made of polyimide, providing excellent thermal isolation and high mechanical compliance. Electrical interconnect from the substrate to the thermal probes is embedded within the polyimide. The cantilevers are $180 \mu \mathrm{m}$ long by $60 \mu \mathrm{m}$ wide, the common beam segment is $560 \mu \mathrm{m}$ long by $650 \mu \mathrm{m}$ wide and the probe tips are spaced $85 \mu \mathrm{m}$ apart.

To scan a sample, the thermal probe array is inverted and mounted on a motorized XYZ scanning stage (figure 1). The $\mathrm{Z}$-axis actuation is used to lower the thermal probe array onto the sample and pre-deflect the thermal probes. The amount of pre-deflection together with the surface forces between the tip and sample determines the deepest valley that may be scanned 
without losing contact. The $\mathrm{x}$ - and $\mathrm{y}$-axis actuations are then used to raster the probe array across the sample. The thin film metal resistor on each probe tip is used both to heat the tip and to detect temperature variations that occur during the scan due to the thermal coupling between the thermal probe and substrate. Each probe in the array is connected to a separate Wheatstone bridge that provides the bias current and senses the changes in resistance. A separate low noise amplifier and low pass filter on each probe channel are used to minimize noise. A computer interface is used for data acquisition and to control the position of the xy scanning stage. It is notable that the simplicity of this apparatus is afforded in large part by the mechanical compliance of the probes, which permits them to be operated without force feedback.

There are a number of advantages that arise from the choice of flexible polyimide as the structural material for the probe. First, the Young's modulus of polyimide is about $50 \times$ lower than that of silicon nitride, the conventional material of choice for force microscopy probes. For example, the spring constant of a $3 \mu \mathrm{m}$ thick probe in the array of the dimensions described at the beginning of this section can be estimated as $0.15 \mathrm{~N} \mathrm{~m}^{-1}$, which is an order of magnitude smaller than what is typical of probes for AFM. Thus, with the polyimide probes, a topographical variation of $0.2 \mu \mathrm{m}$ scanned without mechanical feedback causes a variation of $30 \mathrm{nN}$ in the contact force, which is acceptable for a number of applications. (In addition, the tip diameter for thermal probes is larger than for AFM probes, so the contact pressure is lower for the same applied force.) For samples that are more sensitive to contact pressure, longer probe shanks can provide even smaller variations in contact force. Second, the compliant nature of polyimide is also advantageous in terms of its reliability and dynamic range. Using a first order beam analysis, the maximum deflection that causes beam fracture is [11]

$$
w_{\mathrm{MAX}}=\sigma_{T S} \frac{2 L^{2}}{3 E H} \propto \frac{\sigma_{T S}}{E}
$$

where $E$ is the Young's modulus, $\sigma_{T S}$ is the tensile strength of the material and $L$ is the length of the beam. Ignoring cantilever dimensions, a figure of merit for the dynamic range of a structural material is $\sigma_{T S} / E$. Silicon nitride has a Young's modulus of about $270 \mathrm{GPa}$ and will fracture at about $500 \mathrm{MPa}$ tensile stress. Polyimide deforms at a yield stress of $350 \mathrm{MPa}$, but has a Young's modulus of only $8.5 \mathrm{GPa}$. The dynamic range of the polyimide probe, therefore, is over an order of magnitude better than a nitride probe of the same dimensions, allowing for larger topographies to be scanned without damage to the cantilever. This also tends to reduce the overall fragility of the polyimide probes compared to silicon nitride probes. The choice of flexible polyimide is also very helpful in the fabrication and assembly of the probes, as will be discussed in the section on fabrication.

The mechanical operation of the array is depicted in figure $3(a)$. Prior to the scan, the probe array is brought into contact with the sample and the cantilevers are pre-deflected by an amount that is application-specific and user-definable. Ideally, each probe deflects without affecting the other probes. Some degree of coupling is inevitable because, during fabrication, all the probes are simultaneously flipped using a
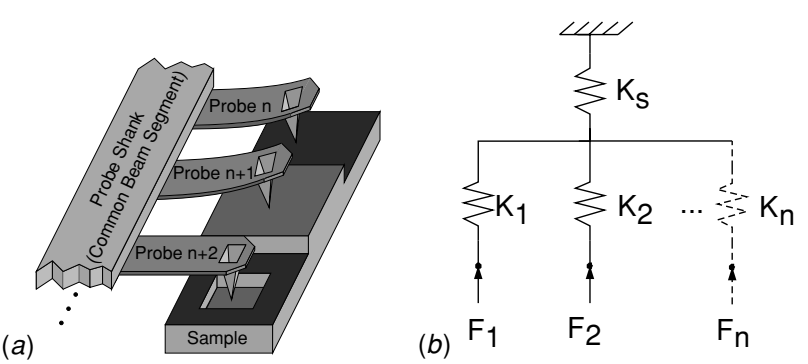

Figure 3. (a) Schematic showing the conceptual operation of the probe array over a sample with large topographic variation. (b) First order mechanical model of the array structure. For effective mechanical decoupling, the spring constant of the shank $K_{S}$ should be large compared to the cantilever spring constants $\mathrm{K}_{1}, \mathrm{~K}_{2}$, etc.

common segment of the beam for easier handling. Intuitively, the system can be thought of as the simple mechanical network shown in figure $3(b)$, with an array of springs representing individual cantilevers coupled to a single spring representing the common beam segment. The precise value of mechanical cross-talk between beams depends on a number of factors, including the length and width of the individual beams and the common segment (and other dimensions), the amount of pre-deflection of the beams when in contact with the sample, and the magnitude of attractive surface forces that exist between each tip and the sample. This attractive force is dependent upon scan conditions, but is typically in the range of $10-100 \mathrm{nN}$ [12].

For proper operation, all the thermal probes in the array must stay in contact with the sample during the scan. However, since the probes are all connected by a common segment, excessive deflection in one probe can cause other probes in the array to lift off the sample. It is worthwhile to know the maximum topographical variation that a single probe can scan over without adjusting adjacent probes off the sample. This is defined as the maximum dynamic range.

Predicting the maximum dynamic range is somewhat challenging because the movement of individual probes causes both bending and twisting of the common beam segment. Since it depends upon the amount of pre-deflection and tipsample adhesion forces, the maximum dynamic range of a single probe is influenced by the instantaneous deflection of every other probe in the array. To illustrate some common situations encountered, finite element analysis (FEA) was performed using ANSYS to determine the dynamic range for scan conditions that will provide a large dynamic range as well as conditions for a small dynamic range. A constant $100 \mathrm{nN}$ force is used to model the attractive forces between the tip and the sample. The dynamic range for varying amounts of predeflection is found by raising probe $\mathrm{A}$ and determining when probe B lifts off the sample. Figure $4(b)$ shows the results of the simulation for adjacent probes at the center of the array (middle line), for probes at the two ends of the array (top line) and for the case where all probes except one are raised (bottom line). From the viewpoint of the designer, it is best to increase the lengths of the separated segments and minimize the length of the common segment. Eventually, the dynamic range becomes limited not by coupling, but by the range of deflection of the cantilever itself. 

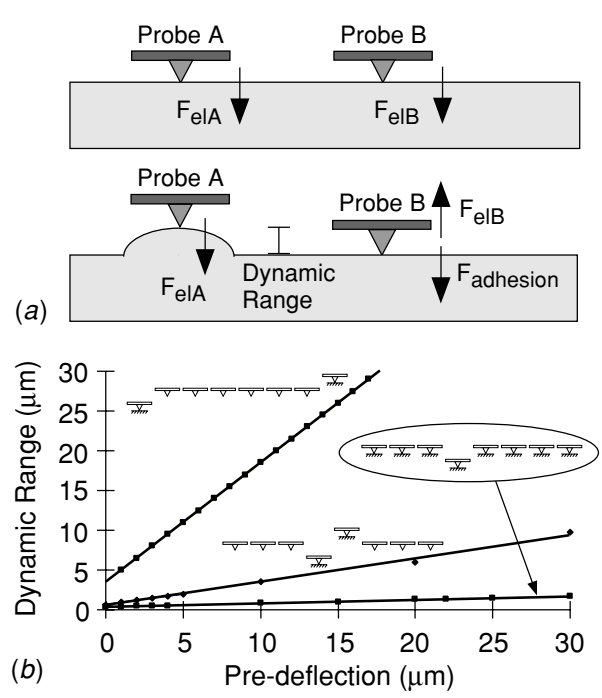

Figure 4. (a) Pre-deflection of the probe array results in elastic forces on adjacent probes $\mathrm{A}$ and $\mathrm{B}\left(\mathrm{F}_{\mathrm{elA}}\right.$ and $\left.\mathrm{F}_{\mathrm{elB}}\right)$. As probe $\mathrm{A}$ moves over a bump, coupling through the common beam segment causes the elastic force exerted on B to reverse direction, and if the reversed force is larger than opposing forces, probe B will lift off the sample, thus limiting the dynamic range. (b) FEA modeling results of the maximum dynamic range as a function of pre-deflection. For all cases, the adhesion force is $100 \mathrm{nN}$.

The thermal coupling ratio between adjacent probes can be defined as $\Delta t_{\mathrm{P} 1} / \Delta t_{\mathrm{P} 2}$, i.e. the temperature shift in a probe caused by a temperature shift in the adjacent probe. Finite element analysis was performed in ANSYS, ignoring convection and assuming that the array is held $15 \mu \mathrm{m}$ above a thermally conductive substrate. The primary path of heat exchange between adjacent probe tips was found to be lateral conduction through the air gap, and the thermal coupling ratio was found to be $-24 \mathrm{~dB}$. The thermal coupling ratio improves by $10 \times$ if the distance between probe tips is doubled to $170 \mu \mathrm{m}$. When the probe tips are in contact with the sample, the cross-talk may increase or decrease depending on the thermal conduction between points of contact and how that compares with conductance through the thickness of the sample.

\section{Fabrication}

The ultracompliant thermal probe array is fabricated with a low temperature 7-mask process suitable for post CMOS fabrication. As shown in figure 5, the process consists primarily of a sequence of surface micromachining steps followed by a flip-over and a thermocompression bonding step to point the tip of the probe away from the substrate.

A pyramidal mold for the tip structure is first created by anisotropic etching of a (100) silicon wafer, masked by a thin layer of silicon nitride (figure $5(a)$ ). To obtain a sharper tip, the silicon is optionally thermally oxidized at $950{ }^{\circ} \mathrm{C}$ [13]. A layer of sacrificial titanium is deposited below the cantilever structures and inside the mold to facilitate the release of the cantilevers (figure 5(b)). The cantilever itself is formed of two polyimide layers (HD Microsystems P2610) with two metal layers embedded within. The first layer of polyimide is etched from the mold region (figure 5(c)). A thin metal layer

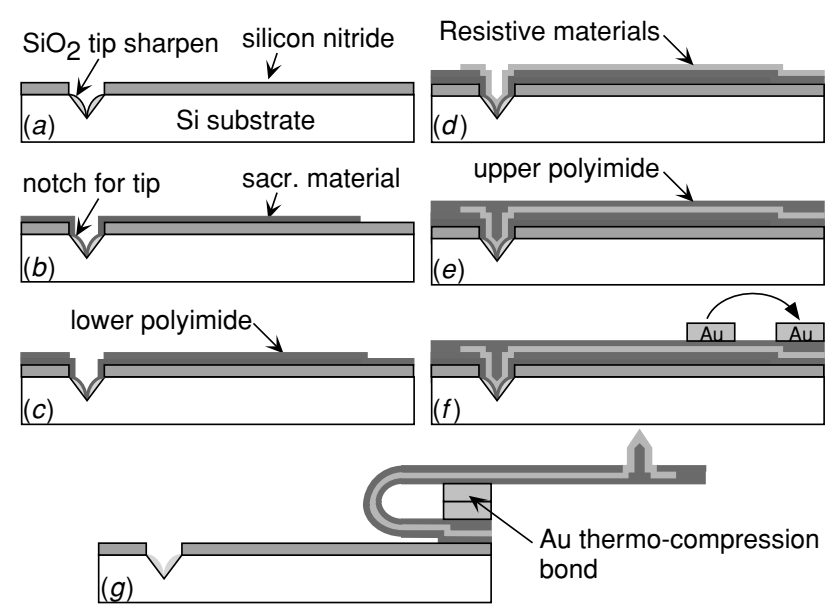

Figure 5. Process flow for fabricating the polyimide-based thermal probe array, suitable for post-IC processing. A tip notch is created, followed by a sacrificial layer, a resistor sandwiched between two layers of polyimide and a gold layer for thermo-compression bonding. After etching the sacrificial layer, the probe is flipped, resulting in a probe overhanging the edge of the die.

consisting of $\mathrm{Cr} / \mathrm{Au}(100 \AA / 1200 \AA)$ is then deposited in the mold to form both the metal tip and the resistor. A second, thicker metal layer consisting of $\mathrm{Cr} / \mathrm{Au} / \mathrm{Cr}(100 \AA / 2500 \AA /$ $100 \AA$ ) forms low-resistance electrical interconnects from the bonding pads to the probe tip (figure $5(d)$ ). The top layer of polyimide is used to insulate the metal and provide strength to the probe tip (figure $5(e)$ ). Thick gold patterns are deposited on top of the second polyimide layer for thermo-compression bonding (figure $5(f)$ ). The cantilevers are released by etching the sacrificial titanium layer in dilute HF. Finally, the probe shank is manually flipped over (for which the mechanical flexibility of the probe shank is essential), and a thermocompression bond is formed at a temperature of $200{ }^{\circ} \mathrm{C}$ (figure $5(g)$ ), resulting in a probe shank overhanging the edge of the wafer die with the probe tip pointed away from the substrate (figure 2). The pre-released device is shown in figure 6.

The final shape of the probe tip, as determined by the (111) planes in a (100) cut Si wafer, is a pyramid of square cross section and $54.7^{\circ}$ sidewalls. The height of the pyramid is approximately $7 \mu \mathrm{m}$. The tip diameter of the probe, which may be rounded somewhat due to processing non-idealities such as deposition characteristics of the sacrificial layer, is typically $<100 \mathrm{~nm}$. As indicated previously, sidewall oxidation of the pyramidal mold reduces this below $50 \mathrm{~nm}$. The entire pyramidal tip is covered with the $\mathrm{Cr} / \mathrm{Au}$ metal layer, which forms the sensing resistor. A thicker layer of the same metals runs back along each probe shank in two parallel lines to large pads. (Adjacent probes may optionally share a pad for the ground line.)

\section{Experimental results and discussion}

The thermal isolation of a single probe in the array was measured while suspended in air (emulating a sample with low thermal conductance) and while in contact with a silicon substrate (emulating a sample with high thermal conductance). 


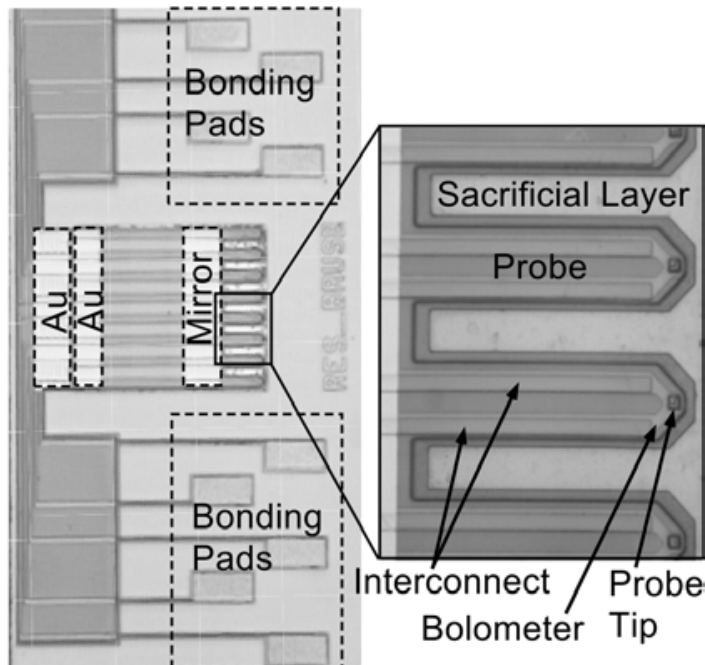

Figure 6. Photograph of the thermal probe array before the flip-over step. The interconnect is a thick metal line to reduce the series resistance to the thin-film bolometer. The two gold regions mate when performing the thermo-compression bond.

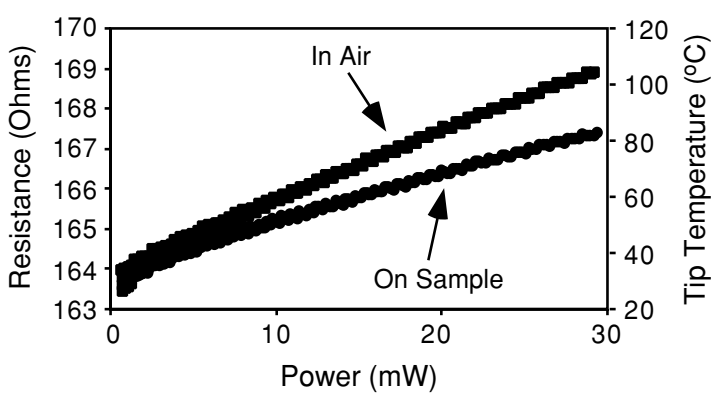

Figure 7. Thermal isolation of a single thermal probe that is suspended in air and in contact with a silicon sample. Probe temperature and resistance increase linearly as input power is ramped.

The thermal isolation depends upon the thermal conductance of the sample with which the probe is in contact. A known current is passed through the thermal probe, the voltage drop across the bolometer is measured and the resistance is plotted as a function of input power (figure 7). Temperature is calculated from resistance using a TCR of $450 \mathrm{ppm}$ (determined in a separate experiment), and the thermal isolation is found to be $2400 \mathrm{~K} \mathrm{~W}^{-1}$ in air and $1700 \mathrm{~K} \mathrm{~W}^{-1}$ when the probe is in contact with a silicon substrate.

To validate the functionality of the probe array, a $750 \mu \mathrm{m} \times 200 \mu \mathrm{m}$ area was scanned on a commercial IC containing bond pads. Four of the eight probes in the array were used, each generating a $200 \mu \mathrm{m} \times 200 \mu \mathrm{m}$ image consisting of 100 lines scanned at $25 \mu \mathrm{m} \mathrm{s}^{-1}$. The raw data were subjected to standard signal processing to remove scan artefacts such as linear trends, and to filter out high frequency noise. The optical image of the scan region and the thermal images obtained from the four probes are shown in figure 8 . The scan results illustrate how a thermal image may be reconstructed by merging overlapping scan regions. The scan, which includes surface variations of up to $2 \mu \mathrm{m}$ (as verified by a line scan taken with a surface profilometer and plotted

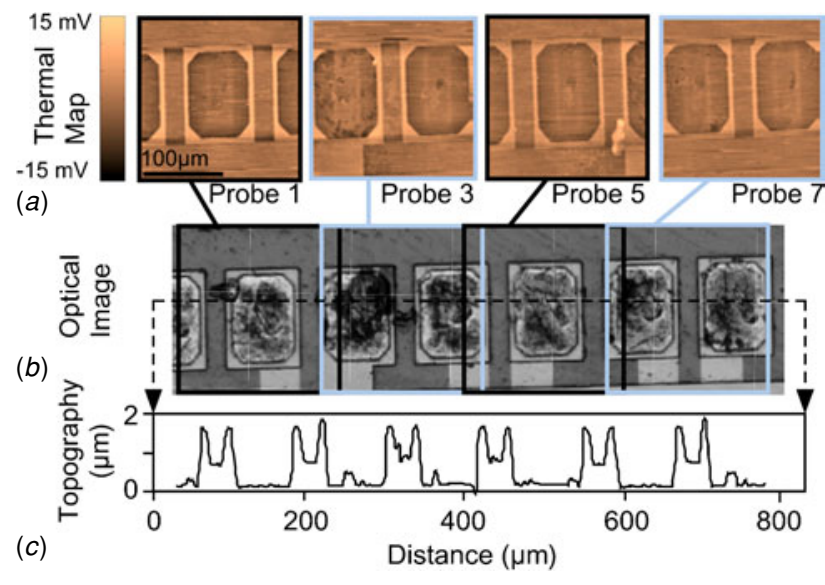

Figure 8. Reconstruction of a thermal image by combining overlapping scan regions. Shown here is a $750 \mu \mathrm{m} \times 200 \mu \mathrm{m}$ area on a commercial IC containing $60 \mu \mathrm{m} \times 85 \mu \mathrm{m}$ bonding pads with $120 \mu \mathrm{m}$ pitch. (a) Thermal images obtained from four probes spaced $170 \mu \mathrm{m}$ apart scanning at $25 \mu \mathrm{m} \mathrm{s}^{-1}$. (b) Optical image. (c) Horizontal line scan taken with a surface profilometer showing $2 \mu \mathrm{m}$ topographical features between bond pads. In this scan, the thermal image conveys primarily topographical information, with sensitivity of $12 \mathrm{mV} \mu \mathrm{m}^{-1}$, dynamic range greater than $2 \mu \mathrm{m}$ and a noise limited minimum detectable topography of $80 \mathrm{~nm}$.
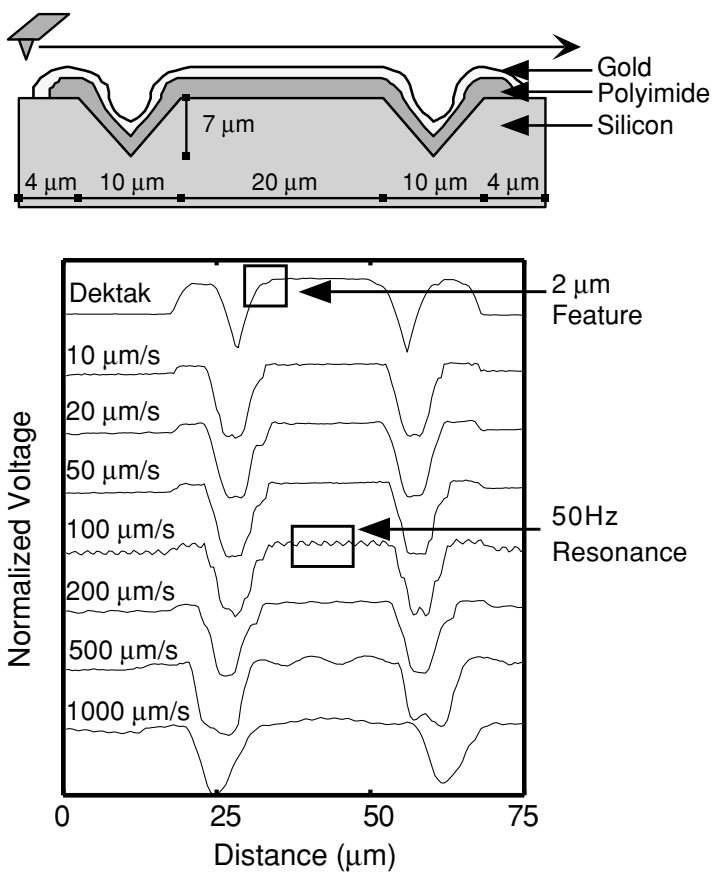

Figure 9. Speed scaling study. Two $7 \mu \mathrm{m}$ pits coated with polyimide and gold were scanned at increasing speeds. A $2 \mu \mathrm{m}$ feature (labeled) is observed at speeds up to $200 \mu \mathrm{m} \mathrm{s}^{-1}$.

Mechanical resonance at $50 \mathrm{~Hz}$ was observed at speeds above $100 \mu \mathrm{m} \mathrm{s}^{-1}$.

in figure $8(c)$ ), is performed without force feedback. This is an example of where the thermal image conveys primarily topographical information. Used in this manner, the probe array can perform parallel, high speed topographical imaging of integrated circuits and surface micromachined MEMS, allowing detection of mechanical defects. However, such an application would probably also require quantitative models that co-relate the images to variations in both topographical 
(a)

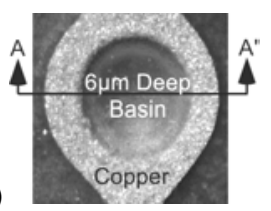

(b)
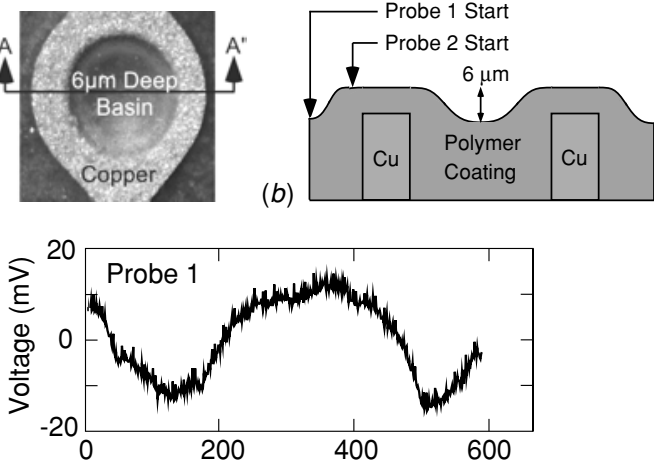

(c)

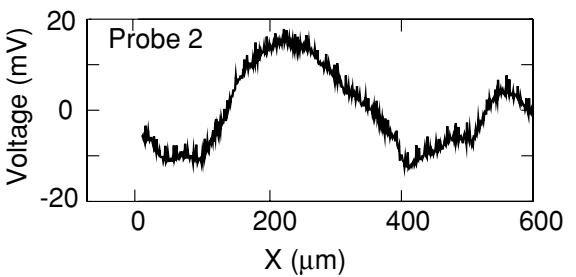

$(d)$
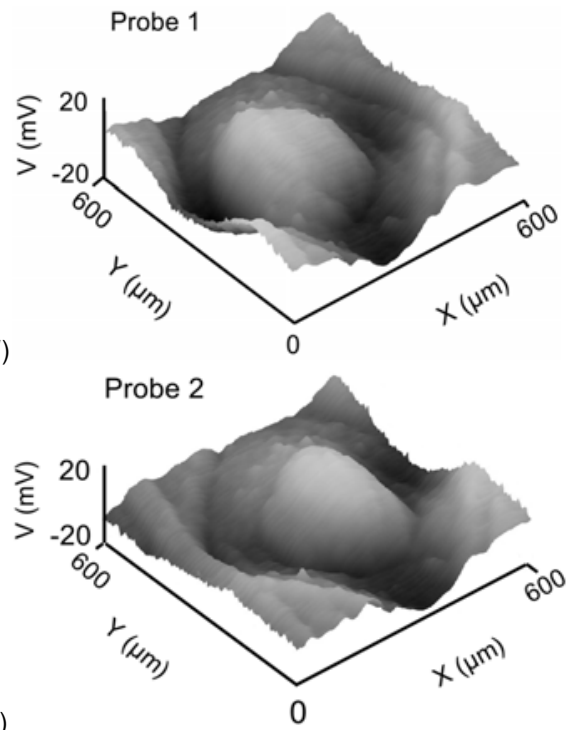

(e)

Figure 10. Mechanical decoupling. (a) Optical image and (b) A-A' cross section of a via with $6 \mu \mathrm{m}$ topography. (c) Line scans taken simultaneously from two thermal probes spaced $85 \mu \mathrm{m}$ apart. $(d),(e)$ Two-dimensional thermal images obtained from the two probes simultaneously.

and thermal properties, as well as standard samples to calibrate these models.

With throughput being the main motivation behind arrayed probes, it is interesting to gauge the scan speed at which features can be resolved. A sample consisting of two $7 \mu \mathrm{m}$ deep anisotropically etched silicon trenches coated with polyimide and gold were scanned at varying speeds. Line scans in figure 9 reveal that a $2 \mu \mathrm{m}$ long feature can be resolved at speeds up to $200 \mu \mathrm{m} \mathrm{s}^{-1}$ and that a $10 \mu \mathrm{m}$ feature can be resolved at a speed up to $2 \mathrm{~mm} \mathrm{~s}^{-1}$. The effective scan speed, which scales with the number of probe tips, is $1600 \mu \mathrm{m} \mathrm{s}^{-1}$ at $2 \mu \mathrm{m}$ resolution for the eight-probe array. This suggests that a $1.5 \mathrm{~mm} \times 1.5 \mathrm{~mm}$ IC with $2 \mu \mathrm{m}$ minimum features can be imaged in approximately $12 \mathrm{~min}$. However, to put this in perspective, it is also necessary to consider dynamic range,

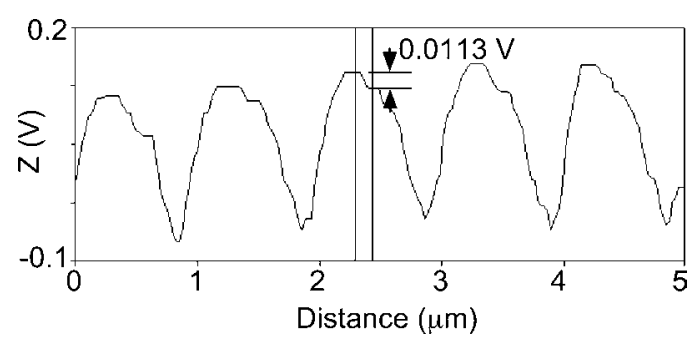

(a)

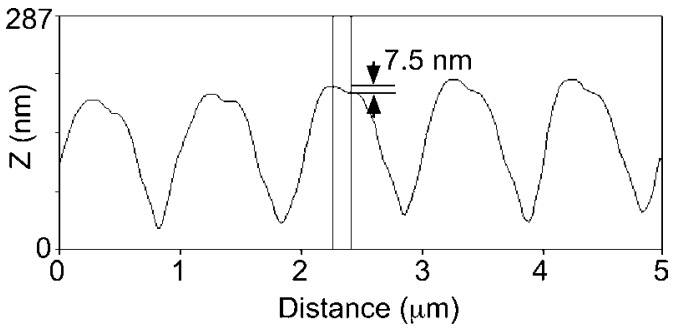

(b)

Figure 11. (a) Lines scan obtained at $10 \mu \mathrm{m} \mathrm{s}^{-1}$ scan speed from a single probe using mechanical feedback control as well as a servo-controlled interface circuit that maintains constant temperature. (b) A topography scan obtained simultaneously with (a), using a reflected laser. This demonstrates that by changing the system parameters and operating conditions, high resolution scans can be obtained from polyimide probes.

because most high throughput thermal probe systems $[4,6]$ are not intended for mapping samples with large topographic variation.

Also demonstrated in figure 9 are dynamic range and topographical resolution. Since this structure is coated with a thin gold layer of uniform thickness, it is expected that the thermal signal obtained will convey primarily topographical characteristics. This is because the primary heat loss from the tip is conduction through the gold layer and across the sample to its underside, which is in contact with a chuck that is uniformly at room temperature [3, 13]. Using a $12 \mathrm{~mA}$ bias current, a $90 \mathrm{mV}$ contrast is obtained over a $7 \mu \mathrm{m}$ topographical variation, indicating a sensitivity of $12 \mathrm{mV} \mu \mathrm{m}^{-1}$. The observed noise voltage is $1 \mathrm{mV}$, translating to a minimum detectable signal (MDS) of approximately $80 \mathrm{~nm}$. The probe, therefore, permits scanning with 6.5-bit resolution over a $7 \mu \mathrm{m}$ dynamic range. This range is $10-100 \times$ higher than typical thermal probe arrays aimed at data storage applications.

Mechanical decoupling between adjacent probes was verified by scanning a via on a printed circuit board that contains a $6 \mu \mathrm{m}$ depression, shown in figure 10 . Two adjacent probes were used simultaneously. Figure 10 shows that the thermal images from the two probes are independent of each other. Although the thermal images are not identical (variation in tip resistance and tip wear can cause differences in the obtained signal), it is clear that the movement of probe 2 into the basin does not affect the signal of probe 1 . Both the line scan and the two-dimensional thermal images support a strong degree of mechanical decoupling over a $12 \mu \mathrm{m}$ topography.

It is instructive to compare the results obtained from the multi-probe array operating without mechanical feedback to 
others obtained from single-shank probes fabricated by the same process. The performance of these single probes using three different kinds of interface circuits: an open-loop circuit using a buffer and a filter (similar to the circuit used for the multi-probe array), a servo-controlled circuit that maintains the probe temperature at a constant user-defined value, and a circuit that dithers the probe temperature and demodulates the output signal to reduce electronic noise are described in $[3,14,15]$. As a point of comparison, figure 11 presents a line scan of a patterned photoresist sample showing submicron lateral resolution. It was obtained at a scan speed of $10 \mu \mathrm{m} \mathrm{s}^{-1}$, using both force feedback as well as electronic temperature control. The compromise in resolution that might occur individually due to the elimination of force feedback, the change in scan speed, the change to a multi-probe geometry, etc, are context dependent. However, comparing the results that are available, there it is evident that probes polyimideshank probes fabricated by the method described are capable of both high speed scanning and high resolution scanning.

\section{Conclusions}

This effort has demonstrated that a mechanically decoupled eight-probe array can be used for parallel, high-resolution thermal mapping of features with large topographical variation without force feedback. Elimination of feedback is enabled by using ultracompliant probes that minimize contact force, preventing damage to both the tip and fragile samples. In non-feedback mode, $2 \mu \mathrm{m}$ lateral features were resolved at scan speeds up to $200 \mu \mathrm{m} \mathrm{s}^{-1}$ per probe, and $10 \mu \mathrm{m}$ features can be resolved even at $2 \mathrm{~mm} \mathrm{~s}^{-1}$. (These tests were performed not to test the resolution of the device but rather, the scanning speeds.) Noise limited MDS for topographical variations was found to be $80 \mathrm{~nm}$, and the dynamic range is $>7 \mu \mathrm{m}$. In comparison, single-shank probes fabricated by the same process can provide lateral spatial resolution $<50 \mathrm{~nm}$ when operated with mechanical servo-control at scan speeds of $10 \mu \mathrm{m} \mathrm{s}^{-1}$ and interfaced with appropriate low-noise circuitry. These results suggest that there are good prospects of obtaining images with high spatial resolution with the multi-probe device.

\section{Acknowledgments}

These devices were fabricated and tested at the SolidState Electronics Laboratory at the University of Michigan. The authors gratefully acknowledge facilities support by the laboratory staff, as well as the assistance of Mr Milind Chinoy in assembling readout circuits.

\section{References}

[1] Williams C C and Wickramsinghe H K 1986 Scanning thermal profiler Appl. Phys. Lett. 49157

[2] Pollock H M and Hammiche A 2001 Micro-thermal analysis: techniques and applications J. Phys. D: Appl. Phys. 34 23-53

[3] Li M H, Lee J H, Menon A K and Gianchandani Y B 2003 Applications of a low contact force polyimide shank bolometer probe for chemical and biological diagnostics Sensors Actuators A $104236-45$

[4] Vettiger P, Despont M, Drechsler U, Durig U, Haberle W, Lutwyche M I, Rothuizen H E, Stutz R, Widmer R and Binnig G K 2000 Millipede-more than one thousand tips for future AFM data storage IBM J. Res. Dev. 44 323-40

[5] Minne S C, Yaralioglu G, Manalis S R, Adams J D, Zesch J, Atalar A and Quate C F 1998 Automated parallel high-speed atomic force microscopy Appl. Phys. Lett. 722340

[6] Lee D W, Ono T, Abe T and Esashi M 2002 Microprobe array with electrical interconnection for thermal imaging and data storage J. Microelectromech. Syst. 11 215-21

[7] Miller S A, Turner K L and MacDonald N C 1997 Scaling torsional cantilevers for scanning probe microscope arrays: theory and experiment Tech. Dig. IEEE Int. Conf. on Solid State Sensors and Actuators (Transducers) (Chicago, IL) pp 455-8

[8] Akiyama T, Staufer U, de Rooij N F, Lange D, Hagleitner C, Brand O, Baltes H, Tonin A and Hidber H R 2000 Integrated atomic force microscopy array probe with metal-oxide-semiconductor field effect transistor stress sensor, thermal bimorph actuator, and on-chip complementary metal-oxide-semiconductor electronics J. Vac. Sci. Technol. B 18 2669-75

[9] Lange D, Akiyama T, Hagleitner C, Tonin A, Hidber H R, Niedermann P, Staufer U, de Rooij N F, Brand O and Baltes H 1999 Parallel scanning AFM with on-chip circuitry in CMOS technology Proc. IEEE Int. Conf. on Micro Electro Mechanical Systems (Orlando, FL) pp 447-52

[10] McNamara S, Basu A, Lee J-H and Gianchandani Y B 2004 Ultracompliant, passively decoupled thermal probe arrays: large area mapping of non-planar surfaces without force feedback Proc. IEEE Int. Conf. on Micro Electro Mechanical Systems (Maastricht, The Netherlands) pp 825-8

[11] Senturia S D 2000 Microsystem Design (Boston, MA: Kluwer)

[12] Majumdar A 1999 Scanning thermal microscopy Annu. Rev. Mater. Sci. 29 505-85

[13] Li M H, Wu J J and Gianchandani Y B 2001 Surface micromachined polyimide scanning thermocouple probes J. Microelectromech. Syst. 10 3-9

[14] Lee J-H and Gianchandani Y B 2004 A scanning thermal probe with servo-controlled interface circuit for microcalorimetry and other applications Rev. Sci. Instrum. 75 1222-7

[15] Lee J-H and Gianchandani Y B 2005 A temperature dithering closed-loop interface circuit for a scanning microscopy system IEEE/ASME J. Microelectromech. Syst. 14 (1) at press 